\title{
Grid-based Gaussian process models for longitudinal genetic data
}

\author{
Wonil Chung ${ }^{1, a, b}$ \\ ${ }^{a}$ Department of Statistics and Actuarial Science, Soongsil University; \\ ${ }^{b}$ Genetic Epidemiology \& Statistical Genetics, Harvard T.H. Chan School of Public Health
}

\begin{abstract}
Although various statistical methods have been developed to map time-dependent genetic factors, most identified genetic variants can explain only a small portion of the estimated genetic variation in longitudinal traits. Gene-gene and gene-time/environment interactions are known to be important putative sources of the missing heritability. However, mapping epistatic gene-gene interactions is extremely difficult due to the very large parameter spaces for models containing such interactions. In this paper, we develop a Gaussian process (GP) based nonparametric Bayesian variable selection method for longitudinal data. It maps multiple genetic markers without restricting to pairwise interactions. Rather than modeling each main and interaction term explicitly, the GP model measures the importance of each marker, regardless of whether it is mostly due to a main effect or some interaction effect(s), via an unspecified function. To improve the flexibility of the GP model, we propose a novel grid-based method for the within-subject dependence structure. The proposed method can accurately approximate complex covariance structures. The dimension of the covariance matrix depends only on the number of fixed grid points although each subject may have different numbers of measurements at different time points. The deviance information criterion (DIC) and the Bayesian predictive information criterion (BPIC) are proposed for selecting an optimal number of grid points. To efficiently draw posterior samples, we combine a hybrid Monte Carlo method with a partially collapsed Gibbs (PCG) sampler. We apply the proposed GP model to a mouse dataset on age-related body weight.
\end{abstract}

Keywords: Bayesian, longitudinal, Gaussian process, hybrid Monte Carlo, PCG Sampler

\section{Introduction}

Many complex traits such as body weight and blood pressure are time-dependent and affected by both genetic as well as nongenetic factors (e.g. age, sex). For these traits, a diversity of statistical methodologies (Ning et al., 2017; Alves et al., 2019) have been developed to map genetic markers, but most identified markers can account for only a small fraction of the estimated genetic variation in these traits (Young, 2019; Chung et al., 2019). Potential sources and research strategies for the missing heritability have been suggested, among which gene-gene and gene-time/environment interactions are of great importance (Clarke and Cooper, 2010; DeWan, 2018). It is known that a single gene may have a small or moderate effect and multiple genes may act jointly. However, most of the current statistical methods only map one gene or two genes jointly since it is computationally prohibitive to map all key

\footnotetext{
This work was supported by the National Research Foundation of Korea (NRF) grant funded by the Korea government (NRF-2020R1C1C1A01012657) and this work was supported by Soongsil University Research Fund.

${ }^{1}$ Corresponding author: Department of Statistics and Actuarial Science, Soongsil University, 369 Sangdo-Ro, DongjakGu, Seoul 06978, Korea. E-mail: wchung@ssu.ac.kr
}

Published 31 January 2022 / journal homepage: http://csam.or.kr

(C) 2022 The Korean Statistical Society, and Korean International Statistical Society. All rights reserved. 
genes associated with complex traits. Moving beyond these limited searches to explore the joint action of genes and time/environment factors would afford us valuable insights into the missing heritability problem.

Several Bayesian methods (Yi, 2004; Banerjee et al., 2008; Chung and Zou, 2014) have been proposed for multiple quantitative trait loci (QTL) mapping. Multiple QTL can be mapped by treating the number of QTL as a random variable (Yi et al., 2002) and alternatively by viewing mapping as a variable selection problem (Yi et al., 2003; Yi, 2004). Bayesian variable selection approaches facilitate the identification of multiple QTL with main and epistasis effects (Yi et al., 2005, 2007; Banerjee et al., 2008; Chung and Zou, 2014). These methods model multiple genes simultaneously and allow for gene-gene interactions and gene-time/environment interactions. However, they only allow for pairwise interactions among genes and time/environment covariates and may miss genes with higherorder interactions. It is not feasible to include all potential effects and their high-order interactions in a parametric model because this can lead to a dramatic increase in the number of parameters and the search space. To overcome this difficulty, we alternatively consider a nonparametric method to search for associated variants without restricting to pairwise interactions among genes and nongenetic factors.

For univariate phenotypes, a nonparametric Bayesian variable selection method with a Gaussian process (GP) prior was developed (Zou et al., 2010), where both genetic and nongenetic effects are modeled nonparametrically. This method was implemented via a hybrid Monte Carlo method and GP prior (Neal, 1996, 1997; Ramussen and Williams, 2006) on the unknown functions for genetic and nongenetic factors. Rather than modeling each main and interaction term explicitly, this GP model measures the importance of each marker, regardless of whether it functions through main effects, epistatic effects or interactions among genes and environment effects non-explicitly. The importance of each genetic and nongenetic factor included in the function is estimated by a single hyperparameter, which enters the covariance function and captures the main and interaction effects associated with each factor. The task is fulfilled by a Bayesian variable selection method through a set of latent indicator variables and gamma mixture priors on both genetic and nongenetic effects.

Another potential factor related to missing heritability is gene-time/environment interaction. To study the time effect, complex traits may be collected on the same subject at multiple time points, resulting in so-called longitudinal data. Although the GP model of Zou et al. (2010) has clear advantages over the current Bayesian gene mapping approaches in detecting high-order interactions, it is not readily applicable to longitudinal data and thus within-subject dependence structures need to be analyzed. Several different approaches are available for approximating within-subject correlation. If the phenotypes are measured at the same time points for all individuals, the records at each time point can be treated as one variable and various statistical approaches have been proposed by treating such longitudinal data as multivariate outcomes (Wu et al., 2002; Ma et al., 2002; Yap et al., 2009). However, if the phenotypes are measured at different time points for some or all individuals, the records cannot be effectively grouped, and thus the multivariate analysis is no longer applicable. Mixed effects models for repeated measures data have become popular in part because they are flexible for unbalanced data (Yang et al., 2006). Furthermore, the existing time-dependent continuity and the correlation of measurements at successive time points can be used to interpolate between adjacent time points. A semiparametric approach for genetic mapping under a mixture-model setting jointly models mean and covariance structures for irregular longitudinal data (Das et al., 2013).

For Bayesian analysis under the mixed effects model framework (Zeger and Karim, 1991; Gilks et al., 1993) where the covariance structure of the random effects is modeled nonparametrically, a large number of parameters are needed which are also constrained by the fact that the covariance matrix 
is nonnegative definite. There is no standard solution to the problem of choosing a prior on the covariance matrix in the mixed effects model (Kass and Natarajan, 2006). The usual inverse-Wishart prior is often inadequate due to its restrictive form on the common degrees of freedom for all the diagonal entries. Moreover, the inverse Wishart prior leads to a strong dependence between variance and correlation: high variance implies high correlation and low variance implies low or moderate correlation. Alternatively, it may be helpful to decompose the covariance matrix into several components and model each component separately. Barnard et al. (2000) worked with a variance-correlation decomposition and Boik (2002) took advantage of a spectral decomposition. Pourahmadi (1999) proposed using a Cholesky decomposition for priors on the covariance matrix, as did Chen and Dunson (2003), whose method has an advantage over the more traditional modified Cholesky decomposition of Pourahmadi (1999) thanks to the total separation of variance and correlation (Pourahmadi, 2007).

In this paper, we extend the nonparametric Bayesian variable selection method with GP prior of Zou et al. (2010) to longitudinal data as we previously described in Chung (2013). It models genetic and time/environment factors via an arbitrary function using a GP prior. To model covariance matrices, we propose a novel grid-based method for data where phenotypes are not measured at a fixed set of times across samples. The grid-based method parsimoniously describes the covariance matrix of each subject with a set of pre-selected time points. For each observation not measured at any of the pre-selected time points, we interpolate it between its two nearest adjacent grid time points. This allows us to model the covariance matrices of all subjects with a common covariance matrix of fixed dimension. We model this common covariance matrix non-parametrically through the modified Cholesky decomposition of Chen and Dunson (2003), which facilitates the use of normal conjugate priors. The average deviance information criterion (DIC) and simplified Bayesian predictive information criterion (BPIC) are proposed for the selection of the optimal number of grid points. To draw posterior samples of parameters, we develop a new Gibbs sampler with improved convergence properties, which is named a hybrid Monte Carlo within partially collapsed Gibbs (PCG) sampler. We conduct numerous simulations and further analyze a mouse dataset on age-related body weight.

\section{Non-parametric Gaussian process (GP) model for longitudinal data}

\subsection{Grid-based Gaussian process (GP) model}

Suppose there are $n$ subjects under study and subject $i$ has its $j^{\text {th }}$ phenotype measured at time $t_{i j}$ $\left(i=1, \ldots, n, j=1, \ldots, n_{i}\right)$. Let $\boldsymbol{x}_{i}^{g}=\left\{x_{i s}^{g}\right\}_{s=1}^{p}$ denote a $p \times 1$ vector of genotypes where $x_{i s}^{g}$ is the $s^{t h}$ marker genotype $(s=1, \ldots, p)$, and $\boldsymbol{x}_{i}^{t}=\left\{x_{i j m}^{t}\right\}_{j=1}^{n_{i}} q$ q denote an $n_{i} \times q$ matrix of nongenetic covariates including time where $x_{i j m}^{t}$ is the $m^{\text {th }}$ covariate $(m=1, \ldots, q)$ measured at time $t_{i j}$. We consider the following GP-based nonparametric Bayesian model,

$$
\boldsymbol{y}_{i}=\boldsymbol{\eta}\left(\boldsymbol{x}_{i}^{g}, \boldsymbol{x}_{i}^{t}\right)+\boldsymbol{p}_{i} \boldsymbol{v}_{i}+\boldsymbol{e}_{i} \quad(i=1, \ldots, n),
$$

where $\boldsymbol{y}_{i}=\left(y_{i 1}, \ldots, y_{i n_{i}}\right)^{T}$ is an $n_{i} \times 1$ vector of phenotypes and $y_{i j}$ is the phenotype value of subject $i$ at time $t_{i j} ; \boldsymbol{\eta}$ is an unknown function which is used to flexibly model genetic and nongenetic effects; $\boldsymbol{e}_{i}$ is an $n_{i} \times 1$ vector of random errors with $\boldsymbol{e}_{i} \sim N_{n_{i}}\left(0, \sigma_{e}^{2} \boldsymbol{I}_{n_{i}}\right)$. To model the correlation among the repeated measurements of the same individual, we partition the observed time interval at $k$ grid points, $\boldsymbol{t}=\left(t_{1}, \ldots, t_{k}\right)^{T}$, and define $\boldsymbol{v}_{i}$ as a $k \times 1$ vector of random effects at the $k$ pre-specified grid time points such that $\boldsymbol{v}_{i} \sim N(0, \boldsymbol{D})$ where $\boldsymbol{D}$ is a $k \times k$ covariance matrix. The matrix $\boldsymbol{p}_{i}=\left(\boldsymbol{p}_{i 1}^{T}, \ldots, \boldsymbol{p}_{i n_{i}}^{T}\right)^{T}$ is defined as follows via a linear interpolation. If $t_{i j}$ (the $j^{\text {th }}$ measurement of subject $i$ ) falls in between the grid points $t_{s}$ and $t_{s+1}\left(t_{s} \leq t_{i j} \leq t_{s+1}\right)$, we let $\boldsymbol{p}_{i j}=\left(\mathbf{0}_{(s-1)}^{T}, t_{s+1}-t_{i j} / t_{s+1}-t_{s}, t_{i j}-t_{s} / t_{s+1}-t_{s}, \mathbf{0}_{(k-s-1)}^{T}\right)$. If 
$t_{i j}=t_{s}$, then $\boldsymbol{p}_{i j}=\left(\mathbf{0}_{(s-1)}^{T}, 1, \mathbf{0}_{(k-s)}^{T}\right)$ and thus if individual $i$ has observations that are only measured exactly at the $k$ grid time points, then $\boldsymbol{p}_{i}$ becomes an identity matrix.

For the covariance matrix $\boldsymbol{D}$, we employ the modified Cholesky decomposition of Chen and Dunson (2003). That is, we let $\boldsymbol{D}=\boldsymbol{L} \boldsymbol{L}^{T}$ where $\boldsymbol{L}$ denotes a $k \times k$ lower triangular Cholesky decomposition matrix that has nonnegative diagonal elements. Further, we let $\boldsymbol{L}=\boldsymbol{\Delta} \boldsymbol{\Psi}$ where $\boldsymbol{\Delta}=\operatorname{diag}\left(\delta_{1}, \ldots, \delta_{k}\right)$ and $\boldsymbol{\Psi}$ is a $k \times k$ matrix with the $(l, m)$ element denoted by $\psi_{l m}$. To make $\boldsymbol{\Delta}$ and $\boldsymbol{\Psi}$ identifiable, we assume $\delta_{l} \geq 0, \quad \psi_{l l}=1$ and $\psi_{l m}=0(l=1, \ldots, k, m=l+1, \ldots, k)$. These conditions lead to $\Delta$ being a nonnegative $k \times k$ diagonal matrix and to $\boldsymbol{\Psi}$ being a lower triangular matrix with all its diagonal elements being 1. This results in the decomposition of $\boldsymbol{D}=\boldsymbol{\Delta} \boldsymbol{\Psi} \boldsymbol{\Psi}^{T} \boldsymbol{\Delta}$. We now reparameterize the model (2.1) as,

$$
\boldsymbol{y}_{i}=\boldsymbol{\eta}\left(\boldsymbol{x}_{i}^{g}, \boldsymbol{x}_{i}^{t}\right)+\boldsymbol{p}_{i} \Delta \boldsymbol{\Delta} \boldsymbol{b _ { i }}+\boldsymbol{e}_{i}(i=1, \ldots, n)
$$

where $\boldsymbol{b}_{i}=\left(b_{i 1}, \ldots, b_{i k}\right)^{T}$ with $b_{i j} \sim N(0,1)$ and $b_{i j} \perp b_{i j^{\prime}}\left(j \neq j^{\prime}\right), j=1, \ldots, k$.

\subsection{Prior specifications}

To complete GP-based Bayesian modeling, we need to specify priors on all the unknown parameters. The Bayesian model contains an unknown function $\boldsymbol{\eta}$ and a set of parameters for random effects. For the unknown function $\boldsymbol{\eta}$, we straightforwardly extend the GP prior proposed in Zou et al. (2010) for both genetic and time-varying nongenetic covariates. For random effects, we adopt the priors in Chen and Dunson (2003). Specifically, we impose independent half normal priors on the diagonal elements of $\boldsymbol{\Delta}$ and normal priors on the lower triangular elements of $\boldsymbol{\Psi}$. Below we provide details.

\subsubsection{Prior on $\eta$}

Let $\boldsymbol{\eta}_{i}=\boldsymbol{\eta}\left(\boldsymbol{x}_{i}^{g}, \boldsymbol{x}_{i}^{t}\right)$ denote an $n_{i} \times 1$ vector of unknown functions of $\boldsymbol{x}_{i}^{g}$ and $\boldsymbol{x}_{i}^{t}$, and $\boldsymbol{\eta}=\left(\boldsymbol{\eta}_{1}^{T}, \ldots, \boldsymbol{\eta}_{n}^{T}\right)^{T}$ denote an $N \times 1$ vector where $N=\Sigma_{i=1}^{n} n_{i}$. We put a GP prior with mean 0 and covariance matrix $\Sigma_{N}$ on $\boldsymbol{\eta}$. That is,

$$
\begin{aligned}
\boldsymbol{\eta} \sim N_{N}\left(0, \boldsymbol{\Sigma}_{N}\right), \text { where } \boldsymbol{\Sigma}_{N}=\left[\Sigma_{i j j^{\prime} j^{\prime}}\right]_{N \times N}, \\
\Sigma_{i j i^{\prime} j^{\prime}}=\operatorname{cov}\left(\eta_{i j}, \eta_{i^{\prime} j^{\prime}}\right)=\xi^{2} \exp \left\{-\sum_{u=1}^{p} \rho_{g u}^{2}\left(x_{i u}^{g}-x_{i^{\prime} u}^{g}\right)^{2}-\sum_{v=1}^{q} \rho_{t v}^{2}\left(x_{i j v}^{t}-x_{i^{\prime} j^{\prime} v}^{t}\right)^{2}\right\},
\end{aligned}
$$

where $\xi^{2}, \rho_{g u}^{2}$ and $\rho_{t v}^{2}$ are hyperparameters. The hyperparameter $\xi^{2}$ defines the vertical scale of variation and affects the magnitude of the exponential part. The hyperparameters $\rho_{g s}^{2}$ and $\rho_{t m}^{2}$ for $s^{t h}$ marker genotype and $m^{\text {th }}$ covariate determine the relevance of the various input variables such as genetic and nongenetic covariates. Large values of $\rho_{g s}^{2}$ and $\rho_{t m}^{2}$ indicate that variables $\tilde{\boldsymbol{x}}_{s}^{g}$ and $\tilde{\boldsymbol{x}}_{m}^{t}$ where $\tilde{\boldsymbol{x}}_{s}^{g}=\left(x_{1 s}^{g}, \ldots, x_{n s}^{g}\right)^{T}$ and $\tilde{\boldsymbol{x}}_{m}^{t}=\left(x_{11 m}^{t}, \ldots, x_{n n_{n} m}^{t}\right)^{T}$ are of high importance to the phenotype.

\subsubsection{Priors on $\tau$ and $\gamma$}

Let $\tau_{g s}=1 / \rho_{g s}^{2}$ and $\tau_{t m}=1 / \rho_{t m}^{2}$. We conduct Bayesian variable selection by imposing Gamma mixture priors on the parameters $\tau_{g s}$ and $\tau_{t m}$ (Zou et al., 2010). In order to estimate the posterior inclusion probability, we introduce the latent variables $\gamma_{g s}$ and $\gamma_{t m}$ to indicate which factors (genetic and nongenetic effects) are relevant $\left(\gamma_{g s}, \gamma_{t m}=1\right)$, or irrelevant $\left(\gamma_{g s}, \gamma_{t m}=0\right)$ to the phenotype. We include the latent indicators, $\gamma_{g s}, \gamma_{t m}$ as parameters in the sampling algorithm, which has the benefit of producing a stream of samples $\left\{\gamma_{g s}\right\},\left\{\gamma_{t m}\right\}$ from their marginal posterior inclusion probabilities. 
The posterior inclusion probability for each factor is estimated as the proportion of times the sampler visits the inclusion model $\left(\gamma_{g s}, \gamma_{t m}=1\right)$. Specifically, the Gamma mixture priors for the parameters related to the genetic covariates are given by,

$$
\begin{aligned}
& P\left(\gamma_{g s}=1\right)=1-P\left(\gamma_{g s}=0\right)=p_{g s}, \quad p_{g s} \sim B e\left(p_{g s} \mid a_{g \gamma}, b_{g \gamma}\right), \\
& \tau_{g s} \mid \gamma_{g s} \sim\left(1-\gamma_{g s}\right) G a\left(\tau_{g s} \mid \frac{\alpha_{g 0}}{2}, \frac{\alpha_{g 0}}{2 \mu_{g 0}}\right)+\gamma_{g s} G a\left(\tau_{g s} \mid \frac{\alpha_{g 1}}{2}, \frac{\alpha_{g 1}}{2 \mu_{g 1}}\right),
\end{aligned}
$$

where $\mathrm{Ga}(\tau \mid a, b)$ is the Gamma density $\tau^{a-1} \exp (-b \tau) b^{a} / \Gamma(a)$ and $B e(p \mid a, b)$ is the Beta density $p^{a-1}(1-p)^{b-1} / B(a, b)$. Similarly, the Gamma mixture priors for the parameters related to the nongenetic covariates are,

$$
\begin{aligned}
& P\left(\gamma_{t m}=1\right)=1-P\left(\gamma_{t m}=0\right)=p_{t m}, \quad p_{t m} \sim B e\left(p_{t m} \mid a_{t \gamma}, b_{t \gamma}\right), \\
& \tau_{t m} \mid \gamma_{t m} \sim\left(1-\gamma_{t m}\right) G a\left(\tau_{t m} \mid \frac{\alpha_{t 0}}{2}, \frac{\alpha_{t 0}}{2 \mu_{t 0}}\right)+\gamma_{t m} G a\left(\tau_{t m} \mid \frac{\alpha_{t 1}}{2}, \frac{\alpha_{t 1}}{2 \mu_{t 1}}\right),
\end{aligned}
$$

respectively. Here, $\alpha_{g 0}, \alpha_{g 1}, \alpha_{t 0}$ and $\alpha_{t 1}$ are positive shape parameters and $\mu_{g 0}, \mu_{g 1}, \mu_{t 0}$ and $\mu_{t 1}$ are the means of the Gamma distributions in equation (2.5) and equation (2.6), respectively. Let $\mu_{g 0}=c_{g}^{2} \mu_{g 1}$ and $\mu_{t 0}=c_{t}^{2} \mu_{t 1}$. If we set $c_{g}^{2}$ (or $c_{t}^{2}$ ) to a large value, $\mu_{g 0}\left(\right.$ or $\left.\mu_{t 0}\right)$ is large. In this case, when $\gamma_{g s}=0$ (or $\gamma_{t m}=0$ ), we let $\tau_{g s}$ (or $\tau_{t m}$ ) be large and thus the corresponding variable is irrelevant. When $\gamma_{g s}=1$ (or $\gamma_{t m}=1$ ), we let $\tau_{g s}\left(\right.$ or $\tau_{t m}$ ) take on a small value, indicating the corresponding variable is important.

Let $\tau_{\xi}=1 / \xi^{2}, \tau_{e}=1 / \sigma_{e}^{2}$. We let the prior distributions of the two parameters be Gamma distributions with the following densities,

$$
\begin{gathered}
\tau_{\xi} \sim G a\left(\frac{\alpha_{\xi}}{2}, \frac{\alpha_{\xi}}{2 \mu_{\xi}}\right), P\left(\tau_{\xi}\right)=\frac{\tau_{\xi}^{\frac{\alpha_{\xi}}{2}-1}}{\Gamma\left(\frac{\alpha_{\xi}}{2}\right)}\left(\frac{\alpha_{\xi}}{2 \mu_{\xi}}\right)^{\frac{\alpha_{\xi}}{2}} \exp \left(-\frac{\alpha_{\xi}}{2 \mu_{\xi}} \tau_{\xi}\right), \\
\tau_{e} \sim G a\left(\frac{\alpha_{e}}{2}, \frac{\alpha_{e}}{2 \mu_{e}}\right), P\left(\tau_{e}\right)=\frac{\tau_{e}^{\frac{\alpha_{e}}{2}-1}}{\Gamma\left(\frac{\alpha_{e}}{2}\right)}\left(\frac{\alpha_{e}}{2 \mu_{e}}\right)^{\frac{\alpha_{\xi}}{2}} \exp \left(-\frac{\alpha_{e}}{2 \mu_{e}} \tau_{e}\right) .
\end{gathered}
$$

Here, $\alpha_{\xi}, \alpha_{e}$ are positive shape parameters and $\mu_{\xi}, \mu_{e}$ are the means of $\tau_{\xi}$ and $\tau_{e}$, respectively. For later use, we define $\tau=\left(\tau_{g 1}, \ldots, \tau_{g p}, \tau_{t 1}, \ldots, \tau_{t q}, \tau_{\xi}, \tau_{e}\right)^{T}$ and $\gamma=\left(\gamma_{g 1}, \ldots, \gamma_{g p}, \gamma_{t 1}, \ldots, \gamma_{t q}\right)^{T}$.

\subsubsection{Priors on $\boldsymbol{b}, \boldsymbol{\delta}, \boldsymbol{\psi}$}

We let the prior on $b_{i j}$ follow a standard normal distribution, so that the joint prior distribution of the latent variable $\boldsymbol{b}=\left(\boldsymbol{b}_{1}^{T}, \ldots, \boldsymbol{b}_{n}^{T}\right)^{T}$ is $P(\boldsymbol{b}) \stackrel{d}{=} N(\mathbf{0}, \boldsymbol{I})$. In order to specify priors for $\boldsymbol{\Delta}$ and $\boldsymbol{\Psi}$, we define two vectors $\boldsymbol{\delta}=\left(\delta_{l}: l=1, \ldots, k\right)^{T}$ and $\boldsymbol{\psi}=\left(\psi_{m l}: m=2, \ldots, k ; l=1, \ldots, m-1\right)^{T}$. For $\boldsymbol{\delta}$, we assume that the $\delta_{l}$ 's are independent of each other, so $P(\delta)=\Pi_{l=1}^{k} P\left(\delta_{l}\right)$. The prior distribution for $\boldsymbol{\delta}$ is $P(\boldsymbol{\delta})=\Pi_{l=1}^{k} N^{+}\left(\delta_{l} \mid m_{l 0}, s_{l 0}^{2}\right)$ where $N^{+}\left(\delta_{l} \mid m_{l 0}, s_{l 0}^{2}\right)$ is the density of a half normal distribution which is a $N\left(\delta_{l} \mid m_{l 0}, s_{l 0}^{2}\right)$ density truncated below by zero. The prior distribution for $\psi$ is given by $P(\psi) \stackrel{d}{=} N\left(\psi_{0}, \boldsymbol{R}_{0}\right)$ where $\psi_{0}$ and $\boldsymbol{R}_{0}$ are pre-specified hyperparameters. 


\section{Posterior calculation and sampling scheme}

\subsection{Proposed sampling schemes}

We define $\boldsymbol{\theta}$ as the vector of all unknown parameters, such that $\boldsymbol{\theta}=(\boldsymbol{\tau}, \boldsymbol{\eta}, \omega)$ where $\omega=\left(\boldsymbol{b}^{T}, \boldsymbol{\delta}^{T}, \boldsymbol{\psi}^{T}, \boldsymbol{\gamma}^{T}\right)^{T}$. The joint posterior distribution of $\boldsymbol{\theta}$ is given by,

$$
P(\boldsymbol{\theta} \mid \boldsymbol{y}) \propto P(\boldsymbol{y} \mid \boldsymbol{\theta}) P(\boldsymbol{\tau}) P\left(\boldsymbol{\eta} \mid \boldsymbol{\Sigma}_{N}\right) P(\boldsymbol{\omega}),
$$

where $\boldsymbol{y}=\left(\boldsymbol{y}_{1}^{T}, \ldots, \boldsymbol{y}_{n}^{T}\right)^{T}$. In order to sample the hyperparameters $\boldsymbol{\tau}$, Neal (1997) proposed using a hybrid Monte Carlo method, as Gibbs sampling cannot be easily applied due to the difficulties in sampling from the full conditional posteriors and the Metropolis-Hastings method may produce an inefficient random walk. The hybrid Monte Carlo method is a family of Markov Chain Monte Carlo (MCMC) algorithms which merges the Metropolis-Hastings method with sampling techniques based on dynamic systems in physics (Duane et al., 1987). It suppresses random walks by introducing "momentum" variables associated with "position" variables. To make the hybrid Monte Carlo method independent of the scale of the data, drawing random samples from the posterior distribution for $\tau$ is facilitated by expressing it in logarithmic form thanks to the positivity of $\tau$ (Neal, 1997). However, since $\boldsymbol{b}$ and $\boldsymbol{\psi}$ in $\boldsymbol{\omega}$ can be negative, it is not feasible to take the logarithm of all elements of $\boldsymbol{\omega}$ and thus the hybrid Monte Carlo algorithm cannot be applied to $\omega$. It is more appropriate to use Gibbs sampling for drawing $\omega$ because the posterior distribution of $\omega$ is easily derived (see Section 3.3). Suppose we have a set of arbitrary starting values $\left\{\boldsymbol{\tau}^{(0)}, \boldsymbol{\eta}^{(0)}, \omega^{(0)}\right\}$. We first consider the following sampling scheme.

\section{Sampler 1: Hybrid Monte Carlo within Standard Gibbs Sampler}

- Step 1. Draw $\boldsymbol{\tau}^{(t)}$ from $P\left(\tau \mid \boldsymbol{\eta}^{(t-1)}, \boldsymbol{\omega}^{(t-1)}, \boldsymbol{y}\right)$.

- Step 2. Draw $\boldsymbol{\eta}^{(t)}$ from $P\left(\boldsymbol{\eta} \mid \boldsymbol{\tau}^{(t)}, \boldsymbol{\omega}^{(t-1)}, \boldsymbol{y}\right)$.

- Step 3. Draw $\boldsymbol{\omega}^{(t)}$ from $P\left(\boldsymbol{\omega} \mid \boldsymbol{\eta}^{(t)}, \boldsymbol{\tau}^{(t)}, \boldsymbol{y}\right)$.

Sampler 1 is a three-step standard Gibbs Sampler which updates three groups of unknown parameters $(\tau, \eta, \omega)$ sequentially by sampling $\tau$ using the hybrid Monte Carlo method and $\boldsymbol{\eta}$ and $\omega$ using ordinary Gibbs sampling from their full conditionals under the joint posterior distribution. In order to improve the convergence characteristics of the sampler, we propose replacing the full conditional distribution in step 1 with a conditional distribution marginalized with respect to $\boldsymbol{\eta}$. This approach is not new for GP models and has been used in Zou et al. (2010) where the inferences based on the marginalized conditional distribution provide clearly superior results to the inferences based on the full conditional one.

Next, we propose a more efficient sampler referred as a hybrid Monte Carlo within partially collapsed Gibbs (PCG) sampler below.

Sampler 2: Hybrid Monte Carlo within PCG Sampler

- Step 1. Draw $\boldsymbol{\tau}^{(t)}$ from $P\left(\boldsymbol{\tau} \mid \boldsymbol{\omega}^{(t-1)}, \boldsymbol{y}\right)$ where $P(\boldsymbol{\tau} \mid \boldsymbol{\omega}, \boldsymbol{y})=\int P(\boldsymbol{\tau}, \boldsymbol{\eta} \mid \boldsymbol{\omega}, \boldsymbol{y}) d \boldsymbol{\eta}$.

- Step 2. Draw $\boldsymbol{\eta}^{(t)}$ from $P\left(\boldsymbol{\eta} \mid \boldsymbol{\tau}^{(t)}, \boldsymbol{\omega}^{(t-1)}, \boldsymbol{y}\right)$.

- Step 3. Draw $\boldsymbol{\omega}^{(t)}$ from $P\left(\boldsymbol{\omega} \mid \boldsymbol{\eta}^{(t)}, \boldsymbol{\tau}^{(t)}, \boldsymbol{y}\right)$.

Van Dyk and Park (2008) introduced several basic tools that enable us to transform a standard Gibbs sampler into a PCG sampler with the same stationary target distribution but a faster convergence 
rate. In general, substituting a marginalized conditional distribution into a standard Gibbs sampler will alter the transition kernel and the target distribution. However, both can be maintained by performing PCG sampling in a certain order (Van Dyk and Park, 2008). In Sampler 1, we can modify step 1 by drawing $\boldsymbol{\eta}$ jointly with $\boldsymbol{\tau}$ from $P(\boldsymbol{\eta}, \boldsymbol{\tau} \mid \boldsymbol{\omega}, \boldsymbol{y})$ to get $\left(\boldsymbol{\eta}^{*}, \boldsymbol{\tau}^{(t)}\right)$. Here, a superscript "*” means an intermediate quantity that is sampled but is not part of the output of an iteration. If we then repeat Step 2 and Step 3, we end up with a new sampler, which is a trivial generalization of what is considered a standard sampler, with $\boldsymbol{\eta}$ sampled twice during an iteration (Van Dyk and Park, 2008). In the new sampler, note that $\boldsymbol{\eta}$ is drawn instead of conditioned on in the first step. This marginalization is the key to the superior convergence properties of the PCG sampler and it provides the computational advantages over the standard Gibbs sampler (see Theorem 1 in the supplementary materials for more details). This sampler may be inefficient in that it draws $\boldsymbol{\eta}$ twice in each iteration but the transition kernel and the target distribution will be maintained. Since the first draw of $\boldsymbol{\eta}$ is an intermediate quantity that is not conditioned on and not part of the output, removing the redundant draw of $\boldsymbol{\eta}$ alters neither the transition kernel nor the stationary target distribution. The redundant sampling is removed or trimmed from the new sampler. Since all phases preserve the transition kernel and the target distribution of Sampler 1, the resulting Sampler 2 should converge as Sampler 1 does. It furthermore achieves faster convergence than Sampler 1. In the next two subsections, we will describe detailed sampling schemes for each step in the hybrid Monte Carlo within PCG sampler.

\subsection{Hybrid Monte Carlo method}

Below we describe an efficient sampling scheme for the hybrid Monte Carlo algorithm with marginalized distribution for sampling hyperparameters $\tau$. The conditional distribution marginalized with respect to $\boldsymbol{\eta}$ is $P(\boldsymbol{\tau} \mid \boldsymbol{\omega}, \boldsymbol{y}) \propto P(\boldsymbol{y} \mid \boldsymbol{\tau}, \boldsymbol{\omega}) P(\boldsymbol{\tau})$. The marginal likelihood of $\boldsymbol{y} \mid \boldsymbol{\tau}, \boldsymbol{\omega}$ can be expressed by $\boldsymbol{y} \mid \boldsymbol{\tau}, \boldsymbol{\omega} \sim N_{N}(0, \boldsymbol{\Sigma})$ where $\boldsymbol{\Sigma}=\boldsymbol{\Sigma}_{N}+\boldsymbol{p}\left(\boldsymbol{I}_{n} \otimes \boldsymbol{D}\right) \boldsymbol{p}^{T}+1 / \tau_{e} \boldsymbol{I}_{N}$ and $\boldsymbol{p}=\operatorname{diag}\left(\boldsymbol{p}_{1}, \ldots, \boldsymbol{p}_{n}\right)$. Letting $\boldsymbol{v}=$ $\left(\log \left(\tau_{g 1}\right), \ldots, \log \left(\tau_{g p}\right), \log \left(\tau_{t 1}\right), \ldots, \log \left(\tau_{t q}\right), \log \left(\tau_{\xi}\right), \log \left(\tau_{e}\right)\right)^{T}$, the conditional distribution marginalized with respect to $\boldsymbol{\eta}$ can be written as,

$$
P(\boldsymbol{v} \mid \boldsymbol{\omega}, \boldsymbol{y}) \propto P(\boldsymbol{y} \mid \boldsymbol{\tau}, \boldsymbol{\omega}) P(\boldsymbol{v}) \propto(2 \pi)^{-\frac{N}{2}}|\boldsymbol{\Sigma}|^{-\frac{1}{2}} \exp \left(-\frac{1}{2} \boldsymbol{y}^{T} \boldsymbol{\Sigma}^{-1} \boldsymbol{y}\right) P(\boldsymbol{v}) .
$$

The potential energy of the system is defined as,

$$
\begin{aligned}
\mathcal{E}(\boldsymbol{v}) & =-\log P(\boldsymbol{v} \mid \omega, \boldsymbol{y}) \propto \frac{N}{2} \log (2 \pi)+\frac{1}{2} \log |\boldsymbol{\Sigma}|+\frac{1}{2} \boldsymbol{y}^{T} \boldsymbol{\Sigma}^{-1} \boldsymbol{y}-\log P(\boldsymbol{v}), \\
\frac{\partial \mathcal{E}(\boldsymbol{v})}{\partial v_{i}} & =\frac{1}{2} \operatorname{tr}\left(\boldsymbol{\Sigma}^{-1} \frac{\partial \boldsymbol{\Sigma}}{\partial v_{i}}\right)-\frac{1}{2} \boldsymbol{y}^{T} \boldsymbol{\Sigma}^{-1} \frac{\partial \boldsymbol{\Sigma}}{\partial v_{i}} \boldsymbol{\Sigma}^{-1} \boldsymbol{y}-\frac{1}{P(\boldsymbol{v})} \frac{\partial P(\boldsymbol{v})}{\partial v_{i}},
\end{aligned}
$$

where $v_{i}$ is the $i^{t h}$ element of $v$. The kinetic energy of the system is defined as $\mathcal{K}(\phi)=1 / 2 \sum_{i=1}^{p+q+2} \phi_{i}{ }^{2}$, where $\phi$ is a momentum variable which has $p+q+2$ real-valued components, $\phi_{i}$, in one-to-one correspondence with the components of $\boldsymbol{v}$. The total energy $\mathcal{H}$ of the system, which is called the "Hamiltonian" function, is the sum of the kinetic energy $\mathcal{K}$ and the potential energy $\mathcal{E}$, such that $\mathcal{H}(\phi, v)=\mathcal{K}(\phi)+\mathcal{E}(v)$. The dynamical system simulated through a virtual time $t$ is governed by the following Hamilton's differential equations: $d v_{i} / d t=\partial \mathcal{H} / \partial \phi_{i}=\phi_{i}, \quad d \phi_{i} / d t=-\partial \mathcal{H} / \partial v_{i}=$ $-\partial \mathcal{E} / \partial v_{i}$. Since the partial derivative of $\mathcal{E}$ with respect to $v_{i}$ is complicated, the above equation cannot be simulated exactly. We use leapfrog steps to approximate the dynamic system via the following three equations,

(1) $\phi_{i}\left(t+\frac{\epsilon}{2}\right)=\phi_{i}(t)-\frac{\epsilon}{2} \frac{\partial \mathcal{E}}{\partial v_{i}}(v(t))$, 
(2) $v_{i}(t+\epsilon)=v_{i}(t)+\epsilon \phi_{i}\left(t+\frac{\epsilon}{2}\right)$,

(3) $\phi_{i}(t+\epsilon)=\phi_{i}\left(t+\frac{\epsilon}{2}\right)-\frac{\epsilon}{2} \frac{\partial \mathcal{E}}{\partial v_{i}}(v(t+\epsilon))$,

where $\epsilon$ is the step size for discretizing the dynamic system. The step sizes $\epsilon$ are set to the same value for all hyperparameters and are chosen to scale as $\epsilon \propto N^{-1 / 2}$ since the magnitude of the gradients under the posterior are expected to scale roughly as $N^{1 / 2}$ when the prior is vague. Rasmussen (1996) found that $\epsilon=0.5 N^{-1 / 2}$ performs reasonably well. In summary, one iteration of the hybrid Monte Carlo sampling is as follows,

(1) Starting from the $(i-1)^{\text {th }}$ sample $\left(\boldsymbol{v}^{i-1}, \boldsymbol{\phi}^{i-1}\right)$, perform one leap frog step with step size $\epsilon$, resulting in the proposed value $\left(v^{*}, \phi^{*}\right)$,

(2) Accept the proposed value as $\left(\boldsymbol{v}^{i}, \boldsymbol{\phi}^{i}\right):=\left(\boldsymbol{v}^{*}, \boldsymbol{\phi}^{*}\right)$ with the acceptance rate $\min \left(1, \exp \left[\mathcal{H}\left(\boldsymbol{v}^{i-1}, \boldsymbol{\phi}^{i-1}\right)-\right.\right.$ $\left.\left.\mathcal{H}\left(\boldsymbol{v}^{*}, \boldsymbol{\phi}^{*}\right)\right]\right)$; otherwise retain the previous values with negative momenta as $\left(\boldsymbol{v}^{i}, \boldsymbol{\phi}^{i}\right):=\left(\boldsymbol{v}^{i-1},-\boldsymbol{\phi}^{i-1}\right)$,

(3) Update the total energy of the system by perturbing the momenta according to $\phi_{i}:=\alpha \phi_{i}+$ $p_{i} \sqrt{1-\alpha^{2}}$ for all $i$, where $p_{i}$ are randomly sampled from a standard normal distribution and $\alpha$ is set to 0.95 (Rasmussen, 1996).

Since $v$ and $\phi$ are independent of each other, the Gibbs sampling of the momenta (Step 3) allows the hybrid Monte Carlo sampler to explore regions with different values of $\mathcal{H}$. Finally, we can use the sequence $\left\{v_{i} \mid i=1, \ldots, N\right\}$ as the samples generated from the posterior distribution $P(v \mid \omega, \boldsymbol{y})$.

\subsection{Posterior calculation}

For drawing $\boldsymbol{\eta}$ and $\boldsymbol{\gamma}$, we extend the Gibbs sampling algorithm proposed by Zou et al. (2010) straightforwardly. These algorithms are described in the supplementary materials. For the other parameters, we apply the standard Gibbs sampling algorithm. Specifically, since $\boldsymbol{b}, \boldsymbol{\delta}$ and $\boldsymbol{\psi}$ have multivariate normal or half normal priors, their full conditional distributions can be easily derived by their conjugacy properties. The full conditional posterior distributions of $\boldsymbol{b}, \boldsymbol{\delta}$ and $\boldsymbol{\psi}$ are $P\left(\boldsymbol{b} \mid \boldsymbol{\theta}_{-\boldsymbol{b}}, \boldsymbol{y}\right) \stackrel{d}{=} N\left(\boldsymbol{b}^{*}, \boldsymbol{\Sigma}_{b}^{*}\right)$, $P\left(\delta_{l} \mid \boldsymbol{\theta}_{-\delta_{l}}, \boldsymbol{y}\right) \stackrel{d}{=} N^{+}\left(\delta_{l}^{*}, \sigma_{l}^{* 2}\right)$ and $P\left(\boldsymbol{\psi} \mid \boldsymbol{\theta}_{-\psi}, \boldsymbol{y}\right) \stackrel{d}{=} N\left(\boldsymbol{\psi}^{*}, \boldsymbol{\Sigma}_{\psi}^{*}\right)$, respectively, where $\boldsymbol{\theta}_{-f}$ represents all the elements of $\boldsymbol{\theta}$ except $f$. The expressions for $\boldsymbol{b}^{*}, \boldsymbol{\Sigma}_{b}^{*}, \delta_{l}^{*}, \sigma_{l}^{* 2}, \boldsymbol{\psi}^{*}$ and $\boldsymbol{\Sigma}_{\psi}^{*}$ are given in the supplementary materials as well.

\subsection{Choice of the number of grid points}

An important issue with the proposed grid-based GP model is how to efficiently choose the number of grid points, $k$. To select the optimal number of grid points, we evaluate the goodness of the predictive distributions of our GP model. Spiegelhalter et al. (2002) proposed the deviance information criterion (DIC) as DIC $=-2 E_{\boldsymbol{\theta} \mid \boldsymbol{y}}\{\log P(\boldsymbol{y} \mid \boldsymbol{\theta})\}+P_{D}$. The second term of the DIC, $P_{D}$, is the effective number of parameters, which is defined as $P_{D}=-2 E_{\boldsymbol{\theta} \mid \boldsymbol{y}}\{\log P(\boldsymbol{y} \mid \boldsymbol{\theta})\}+2 \log P(\boldsymbol{y} \mid \overline{\boldsymbol{\theta}})$ where $\overline{\boldsymbol{\theta}}$ are the posterior means of $\boldsymbol{\theta}$. Since $P\left(\boldsymbol{y}_{i} \mid \boldsymbol{\theta}\right) \stackrel{d}{=} N\left(\boldsymbol{\eta}_{i}, \boldsymbol{p}_{i} \boldsymbol{D} \boldsymbol{p}_{i}^{T}+\sigma_{e}^{2} \boldsymbol{I}_{n_{i}}\right)$ in model (2.1), DIC is easy to compute using posterior samples. As stated by Robert and Titterington (2002), the same observed data are used twice to construct $P_{D}$, and thus the predictive distribution chosen by DIC overfits the observed data. To overcome this overfitting problem of DIC, Ando (2007) developed the following Bayesian predictive information criterion (BPIC), defined as BPIC $=-2 E_{\boldsymbol{\theta} \mid \boldsymbol{y}}\{\log P(\boldsymbol{y} \mid \boldsymbol{\theta})\}+2 n \hat{b}$ where $\hat{b}$ is the asymptotic bias of the posterior mean of the expected log-likelihood. Under certain mild regularity conditions, 
the bias term can be approximated by $n \hat{b} \approx P_{D}$ (Ando, 2011), leading to the simplified BPIC $=$ $2 E_{\theta \mid y}\{\log P(\boldsymbol{y} \mid \boldsymbol{\theta})\}+2 P_{D}$. Note that the penalty term of the simplified BPIC is twice that of the original DIC. To choose the optimal number of grid points, we first compute both DIC and simplified BPIC with a series of pre-selected numbers of grid points, among which we select the one with the minimum DIC or simplified BPIC score. The proposed methods have been implemented in the R package "gridgp" which was built on top of the C code developed by Zou et al. (2010) (called "original gp" from here on) for univariate trait mapping. The new sampling scheme and data manipulation procedure have been modified for longitudinal data. For the choice of the optimal number of grid points, gridgp provides both DIC and simplified BPIC scores.

\section{Simulation study and real data analysis}

\subsection{Simulation I}

In this section, we evaluate our grid-based GP model for longitudinal data with simulations. We simulated 200 individuals and 151 markers. Each individual had three to seven measures and the total number of observations was 1,000 . We considered six different setups. We first simulated a set of four markers with only main effects plus one time effect (Setup 1). For the $i^{\text {th }}$ individual, the function $\boldsymbol{\eta}_{i}=c_{g 1} \cdot\left(\boldsymbol{x}_{i 1}+\boldsymbol{x}_{i 2}+\boldsymbol{x}_{i 3}+\boldsymbol{x}_{i 4}+\boldsymbol{t}_{i}\right)$, where the $\boldsymbol{x}_{i a}(a=1, \ldots, 4)$ are genotype vectors of the four simulated markers, $c_{g 1}$ is a scale factor used to control all genetic effects of the four markers, and $\boldsymbol{t}_{i}=$ $\left(t_{i 1}, \ldots, t_{i n_{i}}\right)^{T}$. The time covariates $\boldsymbol{t}_{i}$ were randomly generated from a uniform distribution on $[0,1]$. Next, we simulated datasets containing markers that had main effects and one gene-gene interaction (Setup 2), one gene-time interaction (Setup 3), or two gene-time interactions (Setup 4). The simulated functions were $\boldsymbol{\eta}_{i}=c_{g 2} \cdot\left(\boldsymbol{x}_{i 1}+\boldsymbol{x}_{i 2}+\boldsymbol{x}_{i 3} \cdot \boldsymbol{x}_{i 4}+\boldsymbol{t}_{i}\right)$ for Setup 2, $\boldsymbol{\eta}_{i}=c_{g 3} \cdot\left(\boldsymbol{x}_{i 1}+\boldsymbol{x}_{i 2}+\boldsymbol{x}_{i 3}+\boldsymbol{x}_{i 4} \cdot \boldsymbol{t}_{i}\right)$ for Setup 3 and $\boldsymbol{\eta}_{i}=c_{g 4} \cdot\left(\boldsymbol{x}_{i 1}+\boldsymbol{x}_{i 2}+\boldsymbol{x}_{i 3} \cdot \boldsymbol{t}_{i}+\boldsymbol{x}_{i 4} \cdot \boldsymbol{t}_{i}\right)$ for Setup 4. Last, we simulated data which had one three-way genegene interaction (Setup 5), or one four-way gene-gene interaction (Setup 6). The simulated functions were $\boldsymbol{\eta}_{i}=c_{g 5} \cdot\left(\boldsymbol{x}_{i 1} \cdot \boldsymbol{x}_{i 2} \cdot \boldsymbol{x}_{i 3}+\boldsymbol{x}_{i 4}+\boldsymbol{t}_{i}\right)$ for Setup 5 and $\boldsymbol{\eta}_{i}=c_{g 6} \cdot\left(\boldsymbol{x}_{i 1} \cdot \boldsymbol{x}_{i 2} \cdot \boldsymbol{x}_{i 3} \cdot \boldsymbol{x}_{i 4}+\boldsymbol{t}_{i}\right)$ for Setup 6. We varied $c_{g j}(j=1, \ldots, 6)$ in our simulations to ensure that the proposed method has adequate power. For all setups, we considered three grid time points at $(0,0.5,1)$ and set $\delta=\left(\delta_{1}, \delta_{2}, \delta_{3}\right)=(1,1.2,0.8)$ and $\psi=\left(\psi_{21}, \psi_{31}, \psi_{32}\right)=(0.6,0.4,0.6)$, which resulted in $\boldsymbol{v}_{i} \sim N(0, \boldsymbol{D})$ with $\operatorname{diag}(\boldsymbol{D})=(1,1.96,0.97)$ and the lower triangle elements were $\left(d_{21}, d_{31}, d_{32}\right)=(0.72,0.32,0.81)$. We set $\sigma_{e}^{2}=1$. For the analyses, we chose hyperparameters $\alpha_{x 0}=\alpha_{t 0}=\alpha_{x 1}=\alpha_{t 1}=1, \alpha_{\xi}=\alpha_{e}=0.5, c_{x}=c_{t}=100$, and $\mu_{\xi}=\mu_{e}=400$. We also set $a_{x \gamma}=a_{t \gamma}=0.05$ and $b_{x \gamma}=b_{t \gamma}=0.95$, so that the prior probabilities that each variable (genetic marker or nongenetic covariate) was relevant or irrelevant to the phenotype were 0.05 and 0.95 , respectively. The prior distributions for the elements of $\delta$ were chosen to be independent $N^{+}(0,30)$ and the prior distributions for the elements of $\psi$ were independent $N(0,0.5)$. For each simulated data set, the MCMC algorithm performed for $2 \times 10^{5}$ iterations after discarding the first 1000 burn-in iterations. The remaining MCMC samples were further thinned for every 20 iterations, yielding $10^{4} \mathrm{MCMC}$ samples for the posterior analysis.

To display time-dependent changes in phenotype values for different genotypes of simulated data, we drew time-dependent curves by genotype value at the $1^{\text {st }}$ marker having only a main effect and $4^{\text {th }}$ marker interacting with time for Setup 3. Figure S1 clearly shows that the $4^{\text {th }}$ marker controlled the temporal change in phenotype while the 1 st marker had no interaction with time. The upper left panel of Figure 1 displays the posterior mean estimates of the latent variables $\gamma_{g s}$ and $\gamma_{t m}$ for Setup 1 from gridgp with all time points. All markers were detected based on the average marginal posterior probability of inclusion. In order to compare gridgp with the original gp (Zou et al., 2010), we conducted the analysis with a subset of the data with only one measurement for each subject. 

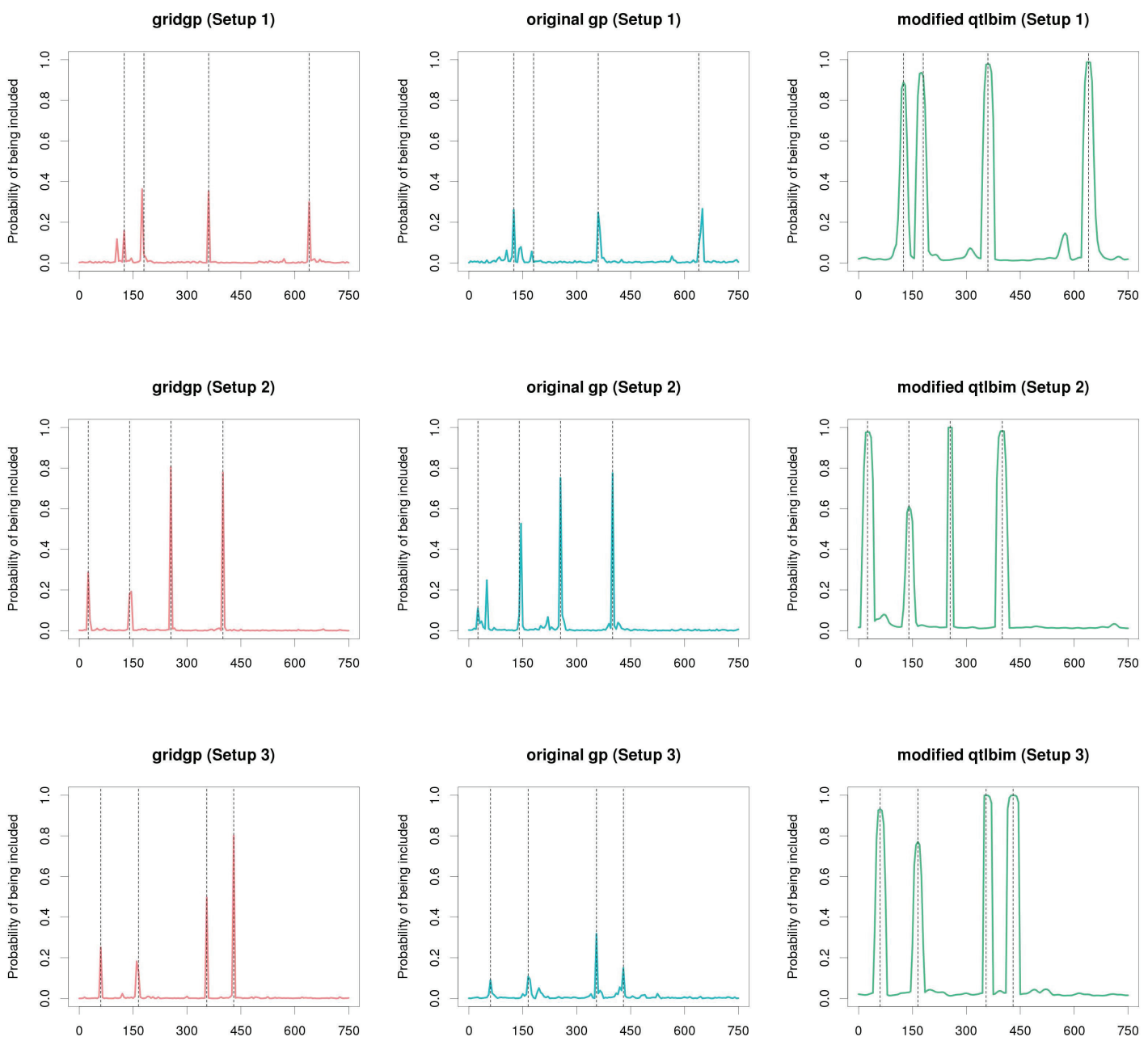

Figure 1: Posterior mean estimates of the latent variables $\gamma_{g k}$ and $\gamma_{t m}$ from gridgp with all time points, original $\mathrm{gp}$ with one randomly selected time point, and modified qtlbim with all time points for Setups 1, 2 and 3.

The results are shown in the upper middle panel of Figure 1. For further comparison, the upper right panel of Figure 1 summarizes the estimated marginal posterior probabilities of each marker for Setup 1 with all time points included from the modified qtlbim, which is a parametric Bayesian multiple association-mapping algorithm for longitudinal data with a built-in variable selection feature (Chung and Zou, 2014). We calculated the marginal posterior probabilities of inclusion based on the Bayes factor (BF) for the modified qtlbim. All markers were identified by the three methods but the marginal posterior inclusion probabilities from gridgp were generally larger than those from the original gp, as shown in Figure 1. The second and third rows of panels in Figure 1 and the top row of Figure 2 summarize the results for Setups 2, 3 and 4, which contained one gene-gene, one gene-time or two gene-time interactions, respectively. All three methods detected the four markers based on the 

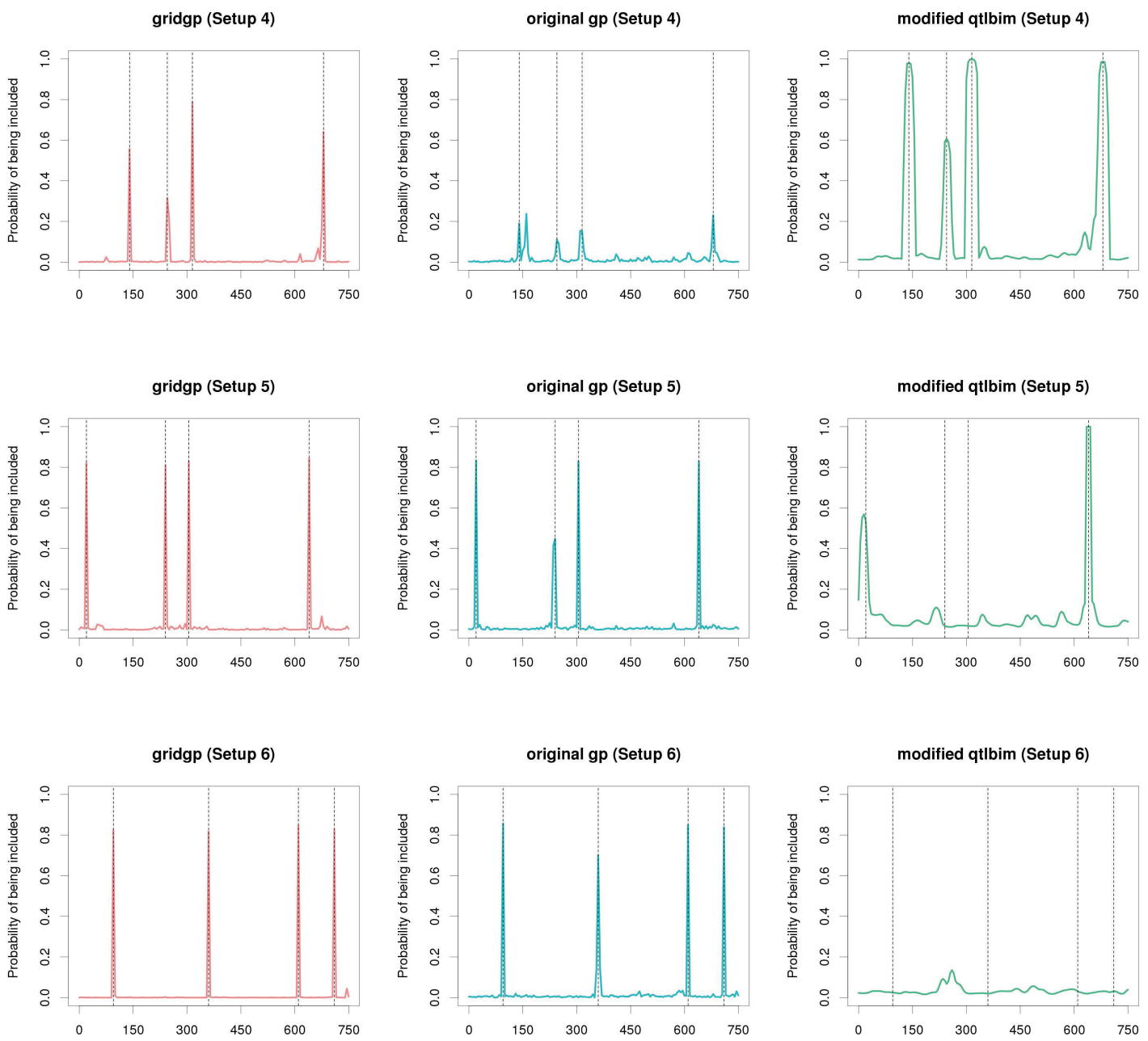

Figure 2: Posterior mean estimates of the latent variables $\gamma_{g k}$ and $\gamma_{t m}$ from gridgp with all time points, original gp with one randomly selected time point, and modified qtlbim with all time points for Setups 4, 5 and 6.

marginal posterior probabilities though the signals from the original gp are smaller than those from gridgp. For Setup 5(one three-way gene-gene interaction) and 6(one four-way gene-gene interaction), both gridgp and the origial gp clearly found all four markers while the modified qtlbim identified only markers with main effects because this parametric model is only capable of detecting main effects and pairwise interactions.

To further compare the methods, we generated the receiver operating characteristic (ROC) curves by varying the cutoffs imposed on the posterior mean and BF, respectively. For each setup, we conducted 100 simulations with uniformly generated marker positions. First, we defined true marker intervals as ones containing one true marker or their two flanking markers (if a true marker is located at one of the two ends, only the single flanking marker is included). For a given cutoff on the pos- 

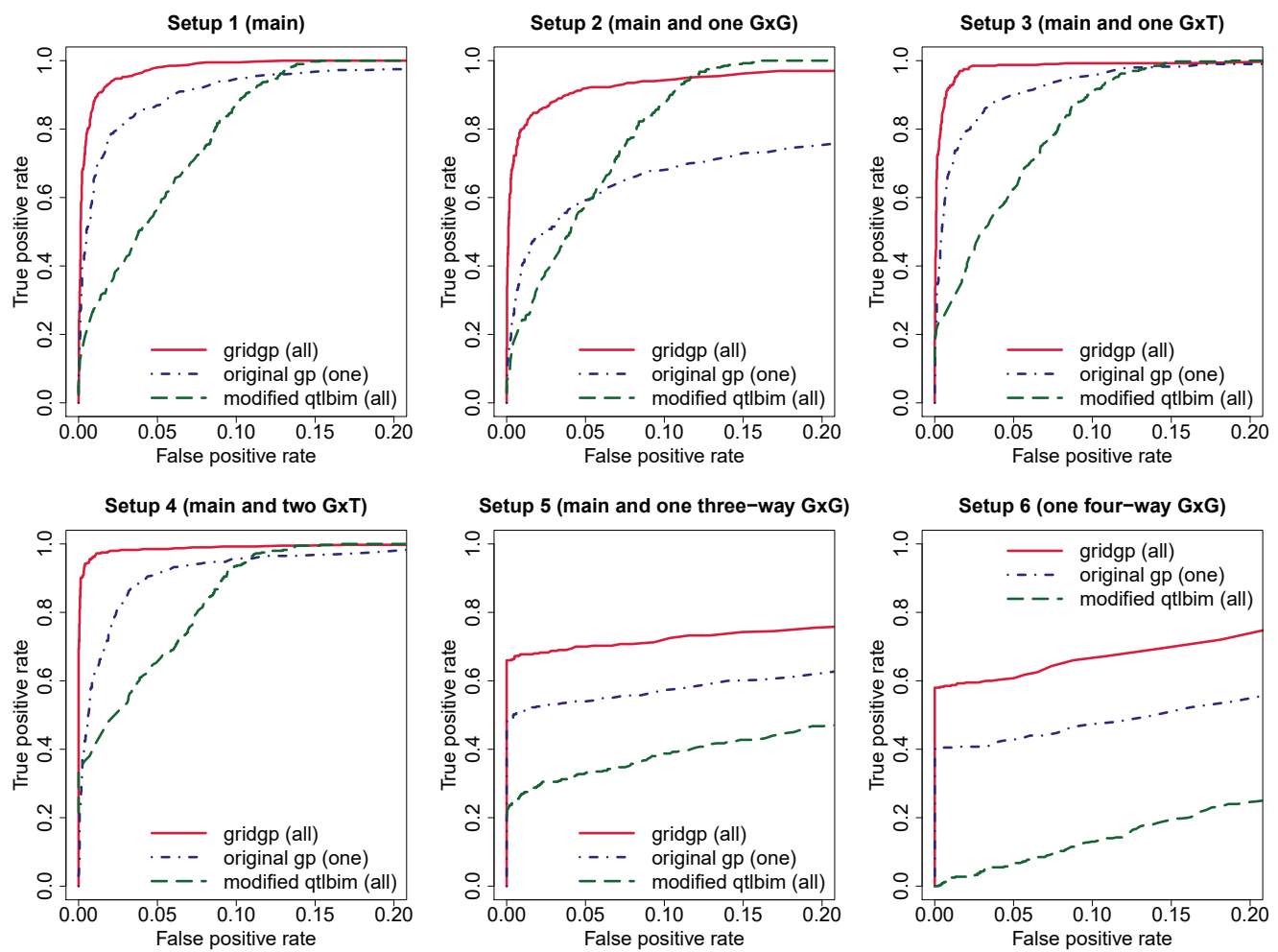

Figure 3: Estimated ROC curves for Setups 1-6: solid lines - proposed gridgp on all data; dot-dashed lines original $g p$ on one time point data; long-dashed lines - modified qtlbim on all data.

terior mean or the $\mathrm{BF}$, a significant interval was defined as one containing at least one marker whose posterior mean or BF exceeded the given cutoff value. A significant interval was classified as a true positive if it was a true marker interval, otherwise it was considered a false positive. We then defined: true positive rate $=(\#$ of significant true intervals $) /(\#$ of true intervals $)$; false positive rate $=(\#$ of significant false intervals)/(\# of false intervals). The ROC curves up to a false positive rate of 0.2 are shown in Figure 3 for all six setups. For Setup 1, gridgp outperformed the modified qtlbim on the full data. The proposed method performed slightly better than the original gp method when the latter was applied to the subset of the data. For Setup 2, the performance of gridgp was similar to the original gp on the subset of the data, and both methods outperformed the modified qtlbim. For Setups 3-6, our new gridgp had higher true positive rates than the other two methods.

In Figure S2, we display the trace plots of $\sigma_{e}^{2}, \delta_{1}, \delta_{2}, \delta_{3}, \psi_{21}, \psi_{31}$ and $\psi_{32}$ for each setup. It shows that all chains moved around the true values for every parameter, indicating good convergence. Figure $\mathrm{S} 3$ presents the marginal posterior and prior densities of the parameters for random errors and random effects. It shows that the distributions of posterior samples look approximately normal around the true values. Table 1 summarizes the posterior estimates of all the parameters for random errors and random effects in the simulation study. The posterior means and medians were close to the true values and all the $95 \%$ highest posterior density (HPD) intervals contained the true values, indicating good performance of our algorithm. 
Table 1: Posterior means, medians, standard deviations and 95\% HPD intervals of the parameters for random errors and random effects in the simulation study

\begin{tabular}{|c|c|c|c|c|c|c|c|c|c|c|c|c|c|}
\hline Setup & Par & True & Mean & Med & SD & $95 \%$ HPD & Setup & Par & True & Mean & Med & SD & $\overline{95 \% \mathrm{HPD}}$ \\
\hline \multirow[t]{7}{*}{1} & $\sigma^{2}$ & 1 & 1 & 1 & 0.03 & $(0.94,1.06)$ & \multirow[t]{7}{*}{2} & $\sigma^{2}$ & 1 & 1 & 1 & 0.03 & $\overline{(0.94,1.07)}$ \\
\hline & $\delta_{1}$ & 1 & 0.96 & 0.96 & 0.15 & $(0.68,1.25)$ & & $\delta_{1}$ & 1 & 1 & 1 & 0.15 & $(0.7,1.3)$ \\
\hline & $\delta_{2}$ & 1.2 & 1.17 & 1.17 & 0.16 & $(0.85,1.47)$ & & $\delta_{2}$ & 1.2 & 1.21 & 1.22 & 0.15 & $(0.91,1.51)$ \\
\hline & $\delta_{3}$ & 0.8 & 0.73 & 0.73 & 0.16 & $(0.43,1.05)$ & & $\delta_{3}$ & 0.8 & 0.72 & 0.72 & 0.16 & $(0.43,1.04)$ \\
\hline & $\psi_{21}$ & 0.6 & 0.61 & 0.58 & 0.25 & $(0.18,1.15)$ & & $\psi_{21}$ & 0.6 & 0.56 & 0.54 & 0.24 & $(0.15,1.08)$ \\
\hline & $\psi_{31}$ & 0.4 & 0.39 & 0.38 & 0.33 & $(-0.21,1.08)$ & & $\psi_{31}$ & 0.4 & 0.49 & 0.48 & 0.34 & $(-0.12,1.2)$ \\
\hline & $\psi_{32}$ & 0.6 & 0.59 & 0.55 & 0.35 & $(0,1.38)$ & & $\psi_{32}$ & 0.6 & 0.63 & 0.59 & 0.34 & $(0.05,1.39)$ \\
\hline \multirow[t]{7}{*}{3} & $\sigma^{2}$ & 1 & 1 & 1 & 0.03 & $(0.94,1.07)$ & \multirow[t]{7}{*}{4} & $\sigma^{2}$ & 1 & 1 & 1 & 0.03 & $\overline{(0.94,1.06)}$ \\
\hline & $\delta_{1}$ & 1 & 0.93 & 0.93 & 0.15 & $(0.64,1.23)$ & & $\delta_{1}$ & 1 & 0.97 & 0.97 & 0.15 & $(0.68,1.28)$ \\
\hline & $\delta_{2}$ & 1.2 & 1.2 & 1.2 & 0.16 & $(0.88,1.5)$ & & $\delta_{2}$ & 1.2 & 1.19 & 1.19 & 0.15 & $(0.88,1.49)$ \\
\hline & $\delta_{3}$ & 0.8 & 0.72 & 0.71 & 0.16 & $(0.41,1.05)$ & & $\delta_{3}$ & 0.8 & 0.7 & 0.69 & 0.16 & $(0.4,1.03)$ \\
\hline & $\psi_{21}$ & 0.6 & 0.55 & 0.53 & 0.25 & $(0.13,1.11)$ & & $\psi_{21}$ & 0.6 & 0.56 & 0.54 & 0.24 & $(0.14,1.1)$ \\
\hline & $\psi_{31}$ & 0.4 & 0.41 & 0.39 & 0.35 & $(-0.25,1.15)$ & & $\psi_{31}$ & 0.4 & 0.46 & 0.45 & 0.35 & $(-0.18,1.2)$ \\
\hline & $\psi_{32}$ & 0.6 & 0.63 & 0.6 & 0.35 & $(0.03,1.42)$ & & $\psi_{32}$ & 0.6 & 0.59 & 0.56 & 0.35 & $(-0.01,1.38)$ \\
\hline \multirow[t]{7}{*}{5} & $\sigma^{2}$ & 1 & 0.99 & 0.99 & 0.03 & $(0.93,1.05)$ & \multirow[t]{7}{*}{6} & $\sigma^{2}$ & 1 & 1 & 1 & 0.03 & $(0.94,1.06)$ \\
\hline & $\delta_{1}$ & 1 & 1.12 & 1.12 & 0.15 & $(0.83,1.42)$ & & $\delta_{1}$ & 1 & 1.39 & 1.39 & 0.17 & $(1.05,1.72)$ \\
\hline & $\delta_{2}$ & 1.2 & 1.21 & 1.21 & 0.16 & $(0.89,1.53)$ & & $\delta_{2}$ & 1.2 & 1.23 & 1.23 & 0.16 & $(0.91,1.55)$ \\
\hline & $\delta_{3}$ & 0.8 & 0.81 & 0.8 & 0.17 & $(0.5,1.14)$ & & $\delta_{3}$ & 0.8 & 0.83 & 0.82 & 0.16 & $(0.52,1.16)$ \\
\hline & $\psi_{21}$ & 0.6 & 0.71 & 0.69 & 0.25 & $(0.28,1.26)$ & & $\psi_{21}$ & 0.6 & 0.94 & 0.91 & 0.27 & $(0.45,1.52)$ \\
\hline & $\psi_{31}$ & 0.4 & 0.63 & 0.61 & 0.34 & $(0.02,1.33)$ & & $\psi_{31}$ & 0.4 & 0.88 & 0.87 & 0.36 & $(0.18,1.63)$ \\
\hline & $\psi_{32}$ & 0.6 & 0.61 & 0.59 & 0.33 & $(0.04,1.34)$ & & $\psi_{32}$ & 0.6 & 0.57 & 0.55 & 0.33 & $(-0.01,1.28)$ \\
\hline
\end{tabular}

Abbreviations: Parameters $=$ Par, True values of parameters=True, Standard Deviation=SD, Highest Posterior

Density $=$ HPD

\subsection{Simulation II}

We conducted another simulation to check the performance of the DIC (Spiegelhalter et al., 2002) and the simplified BPIC (Ando, 2007, 2011) for selecting the optimal number of grid points. The simulation settings were mostly the same as in simulation I except that the true number of grid points now varied from 2 to 4 (i.e., true $k=2,3,4$ ). We simulated 100 datasets containing four markers with main effects for each setup. The simulation setup was $\boldsymbol{y}_{i}=c_{g 1} \cdot\left(\boldsymbol{x}_{i 1}+\boldsymbol{x}_{i 2}+\boldsymbol{x}_{i 3}+\boldsymbol{x}_{i 4}+\boldsymbol{t}_{i}\right)+\boldsymbol{p}_{i} \boldsymbol{v}_{i}+\boldsymbol{e}_{i}$, where the $\boldsymbol{x}_{i j}(j=1,2,3,4)$ are the genotype vectors of the four simulated markers and $\boldsymbol{t}_{i}=\left(t_{i 1}, \ldots, t_{i_{i}}\right)^{T}$ are the times at which the $i^{\text {th }}$ individual has data collected. We set $\left(\delta_{1}, \delta_{2}, \delta_{3}, \delta_{4}\right)=(1,1.2,0.8,0.7)$ and $\left(\psi_{21}, \psi_{31}, \psi_{32}, \psi_{41}, \psi_{42}, \psi_{43}\right)=(0.6,0.4,0.6,0.2,0.4,0.6)$. Table 2 shows the average DIC over 100 simulations, average simplified BPIC scores, and the proportion of simulations correctly choosing the number of grid points. All average DIC and BPIC scores achieved minimums at the true grid point number and the proportions selecting the correct number of grid points were $94 \%, 94 \%$ and $85 \%$ for setups with 2,3 and 4 true grip points using DIC, respectively, and $99 \%, 96 \%$ and $70 \%$ using simplified BPIC, respectively. This shows the usefulness of DIC and simplified BPIC for selecting the true number of grid points in the proposed grid-based GP model.

\subsection{Real data analysis}

To further evaluate the grid-based GP model, we analyzed a mouse dataset on age-related body weight in backcross NZO (New Zealand Obese)/HILt and NON (Nonobese Nondiabetic)/Lt mice (Reifsnyder et al., 2000). To identify diabetes-predisposing markers, diabetes-prone (obese) NZO/HILt mice were outcrossed with non-diabetic (non-obese) NON/Lt mice to generate F1 hybrids. F1 mice of both sexes were backcrossed (reciprocal) with non-diabetic NON/Lt mice, resulting in a continuous distribution 
Table 2: Average DIC, average simplifed BPIC scores and percent selecting the correct number of grid points for nonparametric Bayesian model for different numbers of true grid points

\begin{tabular}{ccccccc}
\hline \hline True $k$ & $k$ & Avg DIC & $(\%)$ \#Sel & Avg Sim BPIC & $(\%)$ \#Sel & Avg $P_{D}$ \\
\hline 2 & 2 & 3293.35 & 94 & 3324.03 & 99 & 30.68 \\
& 3 & 3297.69 & 5 & 3331.71 & 1 & 34.02 \\
& 4 & 3302.01 & 1 & 3339.68 & 3 & 37.67 \\
\hline 3 & 2 & 3355.43 & 0 & 3385.84 & 96 & 30.41 \\
& 3 & 3331.26 & 94 & 3365.56 & 1 & 34.30 \\
& 4 & 3342.78 & 6 & 3380.75 & 2 & 37.98 \\
\hline 4 & 2 & 3362.40 & 0 & 3394.76 & 28 & 32.36 \\
& 3 & 3350.01 & 15 & 3385.68 & 70 & 35.67 \\
\hline
\end{tabular}

Abbreviations: Average DIC scores over 100 replicates=Avg DIC, Average simplified BPIC scores=Avg Sim BPIC, Average $P_{D}=$ Avg $P_{D}$, percent selecting the model with the correct number of grid points $=(\%) \#$ Sel

of body weight in 201 male mice reaching 24 weeks of age. Each mouse had three body weight measurements at weeks 8,16 and 24. After marker selection procedure, a total of 84 microsatellite markers were considered for the analysis. Genomewide scans for body weight were performed in Reifsnyder et al. (2000) using one-way analysis of variance (ANOVA) across the genotype classes at each marker, with significance of the ANOVA F-statistic assessed by permutation. The separate analyses on body weight data from weeks 8, 16 and 24 identified several markers on chromosome 1 . We applied the proposed GP model to these body weight data, considering body weight at 8,16 and 24 weeks as longitudinal measurements and age as a covariate. The number of grid points was set to 3 since DIC and simplified BPIC achieved their minimal scores at $k=3$. Table S1 summarizes the posterior estimates of all the parameters for random errors and random effects in the real data study. The inclusion probabilities for the markers identified as those with main and/or interaction effects in Reifsnyder et al. (2000) are shown in Table S2. The genomewide profile of inclusion probability is presented in Figure 4. The first ranked predictor was the age covariate, and the next ranked predictors were markers D1Mit46, D2Mit120 and D10Mit48; this provides strong activities on chromosomes 1, 2 and 10. To further investigate whether the identified markers acted through their main effects or interactions, a parametric longitudinal analysis was conducted with all possible combinations of the three markers and the age covariate. We fit the data with different covariance structures and selected the heterogeneous autoregressive structure based on the BIC criteria. An Fstatistic was computed from the full model likelihood and the likelihood of the model with one term (the main or interaction term) dropped. All identified markers were marginally significant except one on chromosome 2 (D2Mit120: $F=0.52, P=4.71 * 10^{-1}$ ). We next searched for all possible pairwise and higher-order interactions. We found one significant pairwise interaction between chromosomes 2 and 10 (D2Mit120-D10Mit48: $F=4.60, P=3.24 * 10^{-2}$ ). Interestingly, our GP model identified the marker on chromosome 2 which had only an interaction with the other marker on chromosome 10 but no main effect. No marker-age interaction or three-way interactions were identified. We display time-dependent changes in phenotype for two different genotypes at the three identified markers in Figure S4. There was no strong evidence for a marker interacting with age but we reconfirmed strong main effects on chromosomes 1 and 10 in Figure S4.

\section{Discussion}

In this article, we have developed a nonparametric Bayesian variable selection method with a GP prior for longitudinal genetic data. It models all potential genetic and nongenetic covariates without 


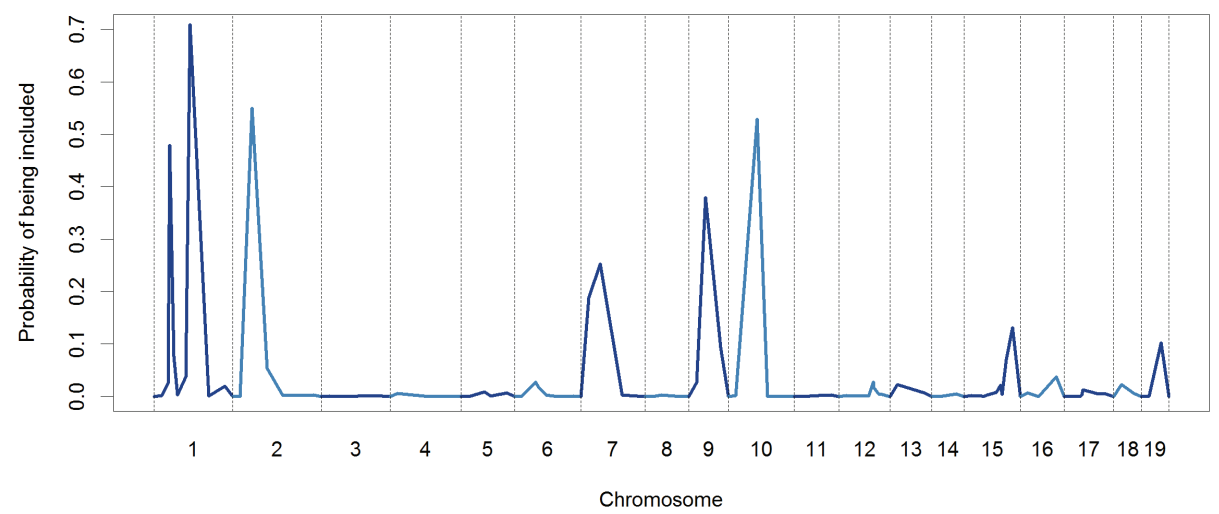

Figure 4: Genomewide profile of probability of being included in the model for body weight in backcross mice involving NZO/HILtJ and NON/ShiLtJ.

restricting to pairwise interactions. Instead of modeling each main and interaction term explicitly, the proposed method measures the importance of each marker, regardless of whether it is mostly due to a main effect or interaction effects via an unspecified function. It is therefore feasible to consider all potential effects and their high-order interactions among genes and other covariates including time. One limitation of the proposed model is its interpretablility. It cannot readily conclude whether the identified markers have main effects and/or interactions with other covariates. Novel findings need to be further evaluated to elucidate their mode of action. If the number of identified genes is small, we may estimate the effect of each genotypic combination, which we can use to interpret the genetic mode.

For the dependence structure of the repeated measurements, we have proposed a grid-based covariance estimation method by employing a modified Cholesky decomposition which provides an unconstrained reparameterization of any covariance matrix. The dimension of the covariance matrix relies on the fixed number of grid points even though subjects may have different numbers of measurements. To approximate the covariance structure, we proposed using a linear interpolation for simplicity. We evaluated other interpolation procedures, such as polynomial or spline methods, and linear interpolation provided comparable performance.

In our simulation, we only utilized 1000 observations, 151 genetic markers and $2 \times 10^{5}$ MCMC iterations, which took approximately an hour. We found that the running time depends linearly on the sample size $(N)$, the number of markers $(P)$ and the number of MCMC iterations $(q)$, which represents $O(N P q)$. To apply the proposed grid-based GP model to large-scale genome-wide association study (GWAS) data, the marker selection or feature selection procedures such as single-maker analysis or linkage disequilibrium (LD) based clumping are currently required due to computation limitations. Our current research focuses on improving the computational feasibility of the proposed grid-based GP model. To enhance the speed of computation, we divide markers into multiple groups and run computations concurrently. We are developing a parallel computing algorithm utilizing message passing interface (MPI), which executes multiple groups of markers simultaneously and synchronizes them at each estimation step, thus making it feasible to apply our method on large-scale GWAS data. When our method is applied to GWAS data, we recommend to include the top principal components (PCs), which are computed from GWAS data, as covariates in the model to account for the population stratification. 
To draw posterior samples, we proposed using the hybrid Monte Carlo within PCG sampler. It is a three-step Gibbs sampler which updates three parameter vectors sequentially by sampling one parameter vector using the hybrid Monte Carlo method and the other two parameter vectors using the ordinary Gibbs sampling approach. This sampler utilizes a marginalized conditional distribution rather than a full conditional distribution in the first sampling step, which improves the convergence properties of the PCG sampler. The conditioning reduction phase does not alter the transition kernel or target distribution of the standard Gibbs sampler. We theoretically investigated the effect of partial collapse of the proposed sampler and concluded that the hybrid Monte Carlo within PCG sampler achieves faster convergence compared to the standard Gibbs sampler.

Another important issue to discuss is convergence of the proposed hybrid Monte Carlo within PCG sampler. As with a standard Gibbs sampler, when one or more draws from the conditionals in a PCG sampler are not available in closed form, such draws are generally implemented by the aid of a Metropolis-Hastings (MH) sampler. However, Van Dyk and Jiao (2014) pointed out that the conditioning reduction in an MH within PCG sampler may change the stationary distribution of the chain and hence care must be taken when MH updates are used in some or all updates of the PCG sampler. For instance, suppose a redundant component that is sampled in one step is again updated according to its full conditional distribution in the following $\mathrm{MH}$ step. To avoid the repeated sampling, the redundant component may be integrated out in the previous step. The following $\mathrm{MH}$ update, however, relies on the component having been integrated out through its acceptance probability (i.e. the MH step depends on the current draw because the proposed draw can be rejected). Therefore, removing the redundant component may alter the stationary distribution in an unpredictable way. In short, an $\mathrm{MH}$ update following a reduction step causes the problem since a reduction step leads to independence that does not exist in the target (Van Dyk and Jiao, 2014). However, this is not the case in the proposed hybrid Monte Carlo within PCG sampler because MH updates in the hybrid Monte Carlo method are used in the reduction step and then standard Gibbs sampling algorithms are conducted in the subsequent steps. We have evaluated the convergence of the proposed sampling scheme with multiple MCMC runs with numerous simulations, and all resulting posterior distributions behave well.

\section{Acknowledgement}

This work was supported by the National Research Foundation of Korea (NRF) grant funded by the Korea government (NRF-2020R1C1C1A01012657) and Basic Science Research Program through the NRF funded by the Ministry of Education (2021R1A6A1A10044154). This work was supported by Soongsil University Research Fund.

\section{References}

Almasy L, Dyer TD, Peralta JM et al. (2014). Data for genetic analysis workshop 18: human whole genome sequence, blood pressure, and simulated phenotypes in extended pedigrees. In $B M C$ proceedings, $\mathbf{8}, 1-9$.

Alves AC, De Silva NMG, Karhunen V et al. (2019). GWAS on longitudinal growth traits reveals different genetic factors influencing infant, child, and adult BMI, Science Advances, 5.

Broman KW, Wu H, Sen Ś, and Churchill GA (2003). R/qtl : QTL mapping in experimental crosses, Bioinformatics, 19, 889-890.

Burton PR, Clayton DG, Cardon LR et al. (2007). Genome-wide association study of 14,000 cases of seven common diseases and 3,000 shared controls, Nature, 447, 661-678. 
Chen Z and Dunson DB (2003). Random effects selection in linear mixed models, Biometrics, 59, $762-769$.

Chung WI, Chen J, Turman C, Lindstrom S, Zhu Z, Loh PR, Kraft P, and Liang L (2019). Efficient cross-trait penalized regression increases prediction accuracy in large cohorts using secondary phenotypes, Nature Communications, 10, 1-11.

Chung WI and Zou F (2014). Mixed-effects models for GAW18 longitudinal blood pressure data. In BMC Proceedings, $\mathbf{8}, \mathrm{S} 87$.

Churchill GA and Doerge RW (1994). Empirical threshold values for quantitative trait mapping, Genetics, 138, 963-971.

Cockerham CC (1954). An extension of the concept of partitioning hereditary variance for analysis of covariances among relatives when epistasis is present, Genetics, 39.

Crow JF and Kimura M (1970). An Introduction to Population Genetics Theory, New York, Evanston and London: Harper \& Row, Publishers.

Das K, Li JA, Wang Z et al. (2011). A dynamic model for genome-wide association studies, Human Genetics, 129, 629-639.

Das K, Li JA, Fu GF, Wang Z, Li RZ, and Wu RL (2013). Dynamic semiparametric Bayesian models for genetic mapping of complex trait with irregular longitudinal data, Statistics in Medicine, 32, 509-523.

Derkach A and Lawless JF, and Sun L (2013). Robust and powerful tests for rare variants using fisher's method to combine evidence of association from two or more complementary tests, Genetic Epidemiology, 37, 110-121.

DeWan AT (2018). Gene-gene and gene-environment interactions, Genetic Epidemiology, 89-110.

Dupuis J and Siegmund D (1999). Statistical methods for mapping quantitative trait loci from a dense set of markers, Genetics, 151, 373-386.

Fisher RA (1918). The correlation between relatives on the supposition of Mendelian inheritance, Transactions of the Royal Society of Edinburgh, 52, 399-433.

Furlotte NA, Eskin E, and Eyheramendy S (2012). Genome-wide association mapping with longitudinal data, Genetic Epidemiology, 36, 463-471.

Gibson G (2012). Rare and common variants: twenty arguments, Nature Reviews Genetics, 13, 135145.

Gilmour AR, Thompson R, Cullis BR (1995). Average information REML: An efficient algorithm for variance parameter estimation in linear mixed models, Biometrics, 1440-1450.

Goodnight CJ (2001). Quantitative trait loci and gene interaction: the quantitative genetics of metapopulations, Heredity, 84, 587-598.

Haley CS and Knott SA (1992). A simple regression method for mapping quantitative trait loci in line crosses using flanking markers, Heredity, 69.

Kamatani Y, Matsuda K, Okada Y, Kubo M, Hosono N, Daigo Y, Nakamura Y, and Kamatani N (2010). Genome-wide association study of hematological and biochemical traits in a Japanese population, Nature Genetics, 42, 210-215.

Kang HM, Sul JH, Service SK, Zaitlen NA, Kong SY, Freimer NB, Sabatti C, and Eskin E (2010). Variance component model to account for sample structure in genome-wide association studies, Nature Genetics, 42, 348-354.

Kang HM, Zaitlen NA, Wade CM, Kirby A, Heckerman D, Daly MJ, and Eskin E (2008). Efficient control of population structure in model organism association mapping, Genetics, 178, 1709_ 1723.

Kao CH and Zeng ZB (1997). General formulas for obtaining the MLEs and the asymptotic variance- 
covariance matrix in mapping quantitative trait loci when using the EM algorithm, Biometrics, 653-665.

Kao CH, Zeng ZB, and Teasdale RD (1999). Multiple interval mapping for quantitative trait loci, Genetics, 152, 1203-1216.

Kao CH and Zeng ZB (2002). Modeling epistasis of quantitative trait loci using Cockerham's model, Genetics, 160, 1243-1261.

Kearsey MJ and Pooni HS (1998). The Genetical Analysis of Quantitative Traits, Stanley Thornes (Publishers) Ltd.

Lander ES and Botstein D (1989). Mapping Mendelian factors underlying quantitative traits using RFLP linkage maps, Genetics, 121, 185-199.

Lee SG, Emond MJ, and Bamshad MJ et al. (2012). Optimal unified approach for rare-variant association testing with application to small-sample case-control whole-exome sequencing studies, American Journal of Human Genetics.

Li B and Leal SM (2008). Methods for detecting associations with rare variants for common diseases: Application to analysis of sequence data, American Journal of Human Genetics, 83, 311-321.

Lippert C, Listgarten J, Liu Y, Kadie CM, Davidson RI, and Heckerman D (2011). Fast linear mixed models for genome-wide association studies, Nature Methods, 8, 833-835.

Ma CX, Casella G, and Wu R (2002). Functional mapping of quantitative trait loci underlying the character process: A theoretical framework, Genetics, 161, 1751-1762.

Madsen BE and Browning SR (2009). A groupwise association test for rare mutations using a weighted sum statistic, PLoS Genetics, 5.

Mather K (1967). Complementary and duplicate gene interactions in biometrical genetics, Heredity, 22,

Mather K and Jinks JL (1977). Introduction to Biometrical Genetics, Cambridge University Press.

Mei H, Chen W, Jiang F et al. (2012). Longitudinal replication studies of GWAS risk SNPs influencing body mass index over the course of childhood and adulthood, PLOS ONE, 7.

Morgenthaler S and Thilly WG (2007). A strategy to discover genes that carry multi-allelic or monoallelic risk for common diseases: A cohort allelic sums test (CAST), Mutation Research/Fund amental and Molecular Mechanisms of Mutagenesis, 615, 28-56.

Morris AP and Zeggini E (2010). An evaluation of statistical approaches to rare variant analysis in genetic association studies, Genetic Epidemiology, 34, 188-193.

Neale BM, Rivas MA, Voight BF et al. (2011). Testing for an unusual distribution of rare variants, PLoS Genetics, 7.

Ning C, Kang HM, Zhou L, Wang D, Wang H, Wang A, Fu J, Zhang S, and Liu J (2017). Performance gains in genome-wide association studies for longitudinal traits via modeling time-varied effects, Scientific reports, 7, 1-12.

Pitman WA, Korstanje R, Churchill GA et al. (2002). Quantitative trait locus mapping of genes that regulate HDL cholesterol in SM/J and NZB/B1NJ inbred mice, Physiological Genomics, 9 , 93-102.

Price AL, Kryukov GV, de Bakker PIW, Purcell SM, Staples J, Wei LJ, Sunyaev SR (2010). Pooled association tests for rare variants in exon-resequencing studies, American Journal of Human Genetics, 86, 832-838.

Purcell S, Neale B, Todd-Brown K et al. (2007). PLINK: A tool set for whole-genome association and population-based linkage analyses, American Journal of Human Genetics, 81, 559-575.

Sabatti C, Service SK, Hartikainen A-L et al. (2009). Genome-wide association analysis of metabolic traits in a birth cohort from a founder population, Nature Genetics, 41, 35-46. 
Sax K (1923). The association of size differences with seed-coat pattern and pigmentation in Phaseolus vulgaris, Genetics, $8,552$.

Schaffner SF, Foo C, Gabriel S, Reich D, Daly MJ, and Altshuler D (2005). Calibrating a coalescent simulation of human genome sequence variation, Genome Research, 15, 1576-1583.

Shang J, Zhang JY, Lei XJ, Zhao WY, and Dong YF (2013). EpiSIM: simulation of multiple epistasis, linkage disequilibrium patterns and haplotype blocks for genome-wide interaction analysis, Genes \& Genomics, 1-12.

Smith EN, Chen W, Kähönen M et al. (2010). Longitudinal genome-wide association of cardiovascular disease risk factors in the Bogalusa heart study, PLoS Genetics, 6.

Strachan DP, Rudnicka AR, Power C et al. (2007). Lifecourse influences on health among British adults: effects of region of residence in childhood and adulthood, International journal of epidemiology, 36, 522-531.

VanLiere JM and Rosenberg NA (2008). Mathematical properties of the $\mathrm{r} 2$ measure of linkage disequilibrium, Theoretical Population Biology, 74, 130-137.

Wu MC, Lee SG, Cai T, Li Y, Boehnke M, and Lin X (2011). Rare-variant association testing for sequencing data with the sequence kernel association test, American Journal of Human Genetics, 89, 82-93.

Wu WR, Li WM, Tang DZ, Lu HR, and Worland, AJ (1999). Time-related mapping of quantitative trait loci underlying tiller number in rice, Genetics, 151, 297-303.

Wu W, Zhou Y, Li W, Mao D, and Chen Q (2002). Mapping of quantitative trait loci based on growth models, TAG Theoretical and Applied Genetics, 105, 1043-1049.

Yandell BS, Mehta T, Banerjee S et al. (2007). R/qtlbim: QTL with Bayesian interval mapping in experimental crosses, Bioinformatics, 23, 641-643.

Yang R, Tian Q, and Xu S (2006). Mapping quantitative trait loci for longitudinal traits in line crosses, Genetics, 173, 2339-2356.

Yap JS, Fan J, and Wu R (2009). Nonparametric modeling of longitudinal covariance structure in functional mapping of quantitative trait loci, Biometrics, 65, 1068-1077.

Yi N, Shriner D, Banerjee S, Mehta T, Pomp D, and Yandell BS (2007). An efficient Bayesian model selection approach for interacting quantitative trait loci models with many effects, Genetics, 176, $1865-1877$.

Young AI (2019). Solving the missing heritability problem, PLoS genetics, 15.

Zeng ZB (1993). Theoretical basis for separation of multiple linked gene effects in mapping quantitative trait loci. In Proceedings of the National Academy of Sciences, 90, 10972-10976.

Zhou X and Stephens M (2012). Genome-wide efficient mixed-model analysis for association studies, Nature Genetics, 44, 821-824. 
